\title{
Preliminary Studies on a Derivative Verotoxin as Oral Adjuvant
}

\author{
Jose Matías ${ }^{1}$, Oscar Landeta ${ }^{2}$, Paula Esquivias ${ }^{2}$ and Carlos Gamazo ${ }^{1^{*}}$ \\ ${ }^{1}$ Department of Microbiology, Institute of Tropical Health (ISTUN), University of Navarra, 31008 Pamplona, Spain \\ ${ }^{2}$ Certest Biotec SL Polígono Industrial Río Gállego II Calle J, 1. 50840 San Mateo de Gállego Zaragoza, Spain
}

*Corresponding author: Carlos Gamazo, Departamento de Microbiología, Universidad de Navarra, 31008 Pamplona, Spain, Tel: +34 948425688 ; Fax: + 3494842 56 49; Email: cgamazo@unav.es

Received date: 16 March 2015; Accepted date: 31 March 2015; Published date: 07 April 2015

Copyright: ( 2015 Matías J, et al. This is an open-access article distributed under the terms of the Creative Commons Attribution License, which permits unrestricted use, distribution, and reproduction in any medium, provided the original author and source are credited.

\begin{abstract}
Most vaccines are still delivered by injection. Mucosal vaccination would increase compliance and decrease the risk of spread of infectious diseases due to contaminated syringes. However, most antigens are unable to induce immune responses when administered mucosally and require the use of strong adjuvant or effective delivery systems. Vibrio cholerae toxin (CT) is a powerful mucosal adjuvants when co-administered with soluble antigens, but present important drawbacks such as residual toxicity. In the current report, a recombinant verotoxin, rVTX1 from Escherichia coli $\mathrm{O} 157$ has been tested to be used as oral adjuvant. A common antigen, BSA (bovine serum albumin), was orally co-administered with the toxoid rVTX1 in BALB/c mice. Commercial CT was used as a reference adjuvant. In this study, the specific antibody response was determined in sera $(\lg G 1, \lg G 2 a, \lg A$ and $\lg E)$ and in fecal samples $(\lg A)$. In addition, the oral toxicity of the new adjuvant candidate was studied in mice. Results indicated that rVTX1 possesses a higher mucosal adjuvant activity than CT when administered orally without inducing any toxic symptoms. These preliminary results support further experiments to demonstrate the potential applications of this protein in oral vaccine development.
\end{abstract}

Keywords: Oral adjuvante; Cholera toxin; Verotoxin; Immunogenicity

\section{Introduction}

Nowadays, the vast majority of vaccines in use are administered by injection, but protective mucosal immune responses are more effectively induced by mucosal immunization such as the oral one [1]. Thus, in contrast to parenteral vaccines, mucosal vaccines induce immunity in both, systemically as well as in mucosal tissues. In addition, mucosal vaccines offer the easiness for administration with the compliance of patients and avoid the use of needles and its subsequent inconveniences. For instance, according to OMS, each year healthcare staff suffers 2 million accidental needle sticks, which $2.5 \%$ results in infection [2]. Despite the many attractive features of oral vaccination, there are some difficulties to be considered: i) chemical barriers such as low $\mathrm{pH}$, digestive enzymes, and surfactant activity by bile salts; ii) physical barriers, such as mucus and epithelial tight junctions [3] and (iii) immune barriers. Certainly, the most difficult problem to solve is the immune tolerance. The mucosal immune system is naturally predisposed to exhibit immune tolerance to the encountered antigens or strange substances in order to prevent unwanted and excessive inflammatory responses [4]. Consequently, potent mucosal adjuvants or antigen delivery systems are needed to overcome natural mucosal tolerance and, then, to promote the induction of mucosal immunity.

Many diverse classes of compounds have been tested as mucosal adjuvants including emulsions, cytokines, polymers, particulate delivery systems and microbial products [5-9]. Nowadays, only six of the vaccines that are currently approved for human use are administered mucosally. These include the live oral vaccines against poliovirus (live attenuated poliovirus strains), Salmonella enterica var.
Typhi (live attenuated S. Typhi), or rotavirus (pentavalent attenuated reassortant rotavirus) [10], and the non-living bacteria of Vibrio cholera adjuvanted with CT (cholera toxin subunit B), Dukoral ${ }^{\circledR}$ [11]. CT has demonstrated adjuvant properties in mucosal vaccines against a variety of diseases [12-17]. However, the clinical use of CT reports several adverse effects such as severe diarrhea after oral administration and the undesirable side effects involving their entry into the central nervous system when given by the nasal route [18]. The CTB subunit lacks the toxicity associated with the CTA subunit, and present strong immunoadjuvant properties. However, this property is reduced when administered orally [19-21].

The benefits derivate from adjuvant incorporation into any vaccine formulation have to be balanced with the risk of adverse reactions and must be justified it is inclusion in the vaccines or therapeutic product $[22,23]$. Thus, efforts are now focused on the development of new mucosal adjuvants that can offer high adjuvant activity with null toxicity. Escherichia coli Shiga toxin strains produce two forms of verotoxin, VTX1 and VTX2. The structure of VTX is described as AB5 (similar to CT), containing one A subunit (the toxic domain) which possess an enzymatic activity, and five B subunits (receptor-binding domain) [24]. The B moiety play a role in the translocation of the A moiety into the cytosol [4].

The aim of this study was to determine if a recombinant form of VTX1 may be used as oral adjuvant. The performed toxicity and immunogenicity studies in BALB/c mice indicated that $\mathrm{rVTX} 1$ possesses a higher mucosal adjuvant activity than CT when administered orally without inducing any toxic symptoms. 


\section{Material and Methods}

\section{Production of toxins and recombinant toxoid}

The cholera toxin was obtained from Sigma-Aldrich (Barcelona, Spain): Native VTX was obtained from a clinical isolated of Escherichia coli $\mathrm{O} 157$ after induction of prophages encoding toxin with a quinolone $[25,26]$. Briefly, E. coli O157 was subcultured in TSB for $4 \mathrm{~h}$ and then treated with $10 \mu \mathrm{g} / \mathrm{ml}$ norfloxacin for prophage induction for $4 \mathrm{~h}$. After inactivation with BEI-FA [27] cells were harvested $(6.000 \mathrm{~g}, 30 \mathrm{~min})$ and the packed cells resuspended in polymyxin $B$ sulfate solution $\left(0.1 \mathrm{mg} / \mathrm{ml}, 37^{\circ} \mathrm{C}, 30 \mathrm{~min}\right)$ to release cell bound toxins, and then filtered using $0.2 \mu \mathrm{m}$ pore size filter. The culture supernatant was precipitated with $70 \%$ ammonium sulfate (12 $\mathrm{h}, 4^{\circ} \mathrm{C}$, under stirring), centrifuged $(16.000 \mathrm{~g}, 10 \mathrm{~min})$ and the pellet was resuspended in PBS and dialized against $10 \mathrm{mM}$ Tris (pH 7.4) for $48 \mathrm{~h}$.

The recombinant VTX1 (rVTX) was cloned and expressed in a pET-30a vector. Briefly, the VTX1 (type strain C600 [H19J]) gene was ligated into the pET-30a expression vector and transformed in BL21 (DE3) strain of E. coli as per standard protocol. Positive clones were selected on LB agar plates containing kanamycin $(50 \mu \mathrm{g} / \mathrm{ml})$. PCR using forward and reverse primers to VTX-1 was used to detect the presence of recombinant plasmid. Positive clones were further confirmed by sequencing analysis before expression of recombinant protein. In order to express the VTX-1, the selected clone was inoculated in $500 \mathrm{ml}$ of $\mathrm{LB}$ broth containing kanamycin $(50 \mu \mathrm{g} / \mathrm{ml})$ and incubated at $37^{\circ} \mathrm{C}, 210 \mathrm{rpm}$. The expression of the recombinant protein was induced by addition of Isopropyl $\beta-D-1$ Thiogalactopyranoside (IPTG) to a final concentration of $1 \mathrm{mM}$, during $4 \mathrm{~h}$. The recombinant protein was purified from the supernatant by affinity chromatography (HisTrapTM HP columns prepacked with precharged $\mathrm{Ni}$ SepharoseTM using the ÄKTAprimeTM automated liquid chromatography system, GE Healthcare).

SDS-PAGE and Immunoblotting analysis: SDS-PAGE was performed using gels of $12 \%$ acrylamide (CriterionTM XT precast gel BIO-RAD). Native and recombinant VTX $(2 \mu \mathrm{g} / \mathrm{mL})$ were treated in sample buffer (10\% SDS, $\beta$-mercaptoethanol) and boiled $10 \mathrm{~min}$ at $100^{\circ} \mathrm{C}$. The gels were stained with Coomassie brilliant blue (BIORAD). The apparent molecular weight of the proteins was determined by comparison with standard molecular weight markers (Rainbow, RPN756, Amersham Pharmacia Biotech).

Unstained proteins separated electrophoretically were transferred from the gel to a nitrocellulose membrane (Whatman ${ }^{\circ}$ Protran $^{\circ}$; pore size $0.45 \mu \mathrm{m}$ ) using a semidry blotting system at $0.8 \mathrm{~mA} / \mathrm{cm} 2$ for 30 min (Trans-Blot ${ }^{\oplus}$ SD Transfer Cell, BIO-RAD). After blocking of protein-binding sites (PBS with 5\% skimmed milk, overnight, RT), the membranes were washed 3 times with PBS-T and then incubated with sera from rabbits immunized with VTX1+VTX2 (Certest Biotech. Zaragoza, Spain) and diluted 1:1,000, $3 \mathrm{~h}$ at $4^{\circ} \mathrm{C}$. The membranes were washed again 3 times with PBS-T and then peroxidase (PO)conjugated secondary antibody (GAR/IgG Fc-PO, 1:1,000) was added for $60 \mathrm{~min}$ at RT. Finally, membranes were extensively washed with PBS- $T$ and the antibody-antigen complexes were visualized after addition of a substrate/chromogen solution $\left(\mathrm{H}_{2} \mathrm{O}_{2} /\right.$ cloro $\alpha$-naftol).

Proteomic analysis: Samples were treated with $1.6 \mu \mathrm{g} / \mathrm{mL}$ of trypsin in $50 \mathrm{mM}$ ammonium bicarbonate at $37^{\circ} \mathrm{C}$ overnight. Tryptic peptides were applied to a MALDI-TOF MS plate in a solution of $10 \mathrm{mg} / \mathrm{mL}$ alpha-cyano-4-hydroxycinnamic acid in $0.1 \%$ trifluoroacetic acid and $50 \%$ acetonitrile. Each digested and desalted sample was resuspended in $10 \mu \mathrm{L}$ of Buffer A (95\% water, $5 \%$ acetonitrile, $0.1 \%$ formic acid) and spotted into a LC-MS/MS system. Peptide mass fingerprints were analysed and searched against theoretical VTX spectra using the Mascot Daemon software package (Matrix Science, London, United Kingdom).The following search parameters were applied: mass accuracy, $\pm 0.25 \mathrm{Da}$; fixed modification of methionine oxidation; and a maximum of one tryptic missed cleavage. All MALDI-TOF MS identifications were performed at least in duplicate. A protein was considered to be identified if the scores from the database searches clearly exceeded the algorithm's significance threshold $(\mathrm{P}<0.05)$.

Toxicity assays: Experiments were performed in compliance with the regulations of the Ethics Committee of the University of Navarra in line with the European legislation on animal experiments. All experiments were carried out in BALB/c female mice, 8-12 weeks old at the onset of the corresponding study.

In vivo mice oral inoculation: Female $\mathrm{BALB} / \mathrm{c}$ mice (6-8 week old; Harlan, Spain) were fasted overnight before inoculation but allowed access to water up to $1 \mathrm{~h}$ before the start of the experiments. Six mice were orally challenged with $40 \mu \mathrm{g}$ of $\mathrm{rVTX} 1$ and monitored for 5 days.

Ligated ileal loop assay: BALB/c mice (6-8 week old; Harlan, Spain) were fasted overnight before inoculation but allowed access to water up to $1 \mathrm{~h}$ before the start of the experiments. The animals were anesthetized by the intraperitoneal administration of $0.2 \mathrm{ml}$ solution of $150 \mathrm{mg} / \mathrm{Kg}$ of Ketamine (Imalgene 1000, (Merial, Barcelona), and 10 $\mathrm{mg} / \mathrm{Kg}$ (Rompum ${ }^{\bullet}$, Bayer Hispania, Barcelona). The abdomen of each mouse was disinfected an antiseptic solution (iodine, Betadine) immediately before surgery. Then, the intestine was exteriorized through a midline incision maintaining a strict aseptic condition. Three intestinal loops ( $1 \mathrm{~cm}$ long) per mouse were prepared by a double ligation of the intestine. Care was taken to avoid interfering with the blood supply. $\operatorname{rVTX} 1(1 \mu \mathrm{g}, 3 \mu \mathrm{g}$, or $5 \mu \mathrm{g}$, in $50 \mu \mathrm{l} \mathrm{PBS})$ was injected into the loop by using a 27 -gauge needle oblique to the intestinal lumen. As a reference, $1 \mu \mathrm{g}$ of CT (in $50 \mu \mathrm{l} \mathrm{PBS}$ ) was inoculated in different loops and animals. Control untreated group was inoculated with $50 \mu \mathrm{l}$ PBS. After inoculation, the incision in the peritoneum, abdominal muscles, and skin was closed in one plane by using super glue. The surgical procedure lasted approximately $10 \mathrm{~min}$ per animal. The animals recovered $30 \mathrm{~min}$ later and they were euthanized $2 \mathrm{~h}$ after surgery. The ligated ileal loops were collected and weighed. Then, the samples were fixed by immersion in formalin for $24 \mathrm{~h}$ before being processed to obtain thin sections $(8 \mu \mathrm{m})$ which were stained with Hematoxylin and eosin for histological analysis.

\section{Oral immunization}

Experiments were performed in compliance with the regulations of the Ethics Committee of the University of Navarra in line with the European legislation on animal experiments. All experiments were carried out in $\mathrm{BALB} / \mathrm{c}$ female mice, 8-12 weeks old at the onset of the priming immunization.

Female BALB/c mice were randomly divided $(n=6)$. Animals were orally immunized with either free BSA (Sigma-Aldrich, ref. A2153) (80 $\mu \mathrm{g}$ or $20 \mu \mathrm{g}$ ) or coadministered with $10 \mu \mathrm{g}$ of either CT or rVTX. The oral immunization was carried out in a total volume of $0.2 \mathrm{ml}$ of PBS by cannula. All animals were sacrificed five weeks after initial immunization. Blood and fecal samples were obtained at weekly intervals after initial immunization until the end of the experiment. 
Serum samples were stored at $-80^{\circ} \mathrm{C}$ until analyzed. Fecal pellets were processed for IgA determination. Briefly, $100 \mathrm{mg}$ of pellets were mixed with PBS containing $0.05 \%$ Tween 20 (PBS-T) plus 3\% non-fatty milk (Sigma-Aldrich). After centrifugation $(10,000 \mathrm{x}$ g, $10 \mathrm{~min})$, supernatants were mixed with protease inhibitor cocktail (SigmaAldrich) and stored at $-80^{\circ} \mathrm{C}$ until analyzed.

Specific antibodies against BSA or the corresponding toxoid in serum and fecal extracts were determined by indirect ELISA. Briefly, microplate wells (Immuno-Maxisorp, Nunc ${ }^{\circledR}$, Roskilde, Denmark) were coated with $10 \mu \mathrm{g} / \mathrm{ml}$ of either BSA or toxoid diluted in sodium carbonate-bicarbonate buffer $(0.05 \mathrm{M}, \mathrm{pH} 9.6)$, and incubated overnight at $4^{\circ} \mathrm{C}$. In case of determination of antibodies against the toxoid, the plates were blocked with PBS containing 3\% BSA for $1 \mathrm{~h}$, RT. Serum or fecal samples (see above) were diluted in PBS (1:80) in triplicates $(1 \mathrm{~h}, \mathrm{RT})$. After intense washing with PBS Tween 20 (PBS$\mathrm{T})$ buffer, the PO-goat anti mouse (classes IgG1, IgG2a, IgA or IgE) (Sigma) was added for $1 \mathrm{~h}$ at $37^{\circ} \mathrm{C}$. The detection reaction was performed by incubating the sample with ABTS substrate for $20 \mathrm{~min}$ at room temperature. Absorbance was measured with an ELISA reader at $405 \mathrm{~nm}$. (Sunrise remote; Tecan-Austria).

\section{Statistical analysis}

All analyses were performed using SPSS $^{\curvearrowleft}$ statistical software for windows. Analysis of variance (ANOVA) was employed to analyze data and, when it indicates a significant difference, the Tukey post hoc test was used to assess the difference between groups. P-values $<0.05$ were considered statistically significant.

\section{RESULTS}

\section{Structural studies of rVTX}

The protein profile of rVTX1was compared with native VTX (nVTX) by SDS-PAGE followed by immunoblotting. The protein staining revealed the presence of a band of $72 \mathrm{kDa}$ which correspond to the holotoxin, and also the subunit A (31-32 kDa), subunit A2 (5 $\mathrm{kDa})$ and the subunit $\mathrm{B}(7.7 \mathrm{kDa})$. Immunoblotting analysis performed with specific sera corroborated the identity of A subunits. The calculated AMW for subunit A in the nVTX1 was $31 \mathrm{kDa}$, whereas in rVTX1was $30 \mathrm{kDa}$. The proteomic analysis revealed a deletion in the subunit A1 of rVTX1 of 6 aminoacids (MHHHHH) equivalent to 820 Da.

\section{Toxicity of VTX1-R and CT toxoids after oral administration}

The mice orally inoculated with $40 \mu \mathrm{g}$ the VTX1-R did not present any symptoms during the follow-up. One of the biological activities of VTX1 and CT is the induction of fluid accumulation in the intestine. Thus a ligated ileal loop assay was carried out in order to measure the toxicity of rVTXland CT by measuring the volume and weight of toxin-induced fluid accumulation in the ligated ileal loops at $3 \mathrm{~h}$ after inoculation. There was a marked fluid accumulation and distention in CT $(1 \mu \mathrm{g})$ treated loops, with an increase of $65 \mu \mathrm{l}$ with respect the PBS control group. In contrast, rVTX1treated loops showed no fluid accumulation with any concentration (Table 1). In order to confirm the results observe from macroscopically analysis, histological studies and subsequent staining was performed. Thin sections of ileal loops 3 h-post inoculation were stained and analyzed under the microscope. Negative control loops were inoculated with PBS, showing normal full-length intestinal villi with a well-preserved epithelium and lamina propria, and no cellular infiltration. Results revealed that $5 \mu \mathrm{g}$ of the rVTX1did not show any cellular infiltration in villi. However, loops inoculated with $1 \mu \mathrm{g}$ of CT exhibited substantial diffuse cellular infiltration of the mucosa and villi (results not shown).

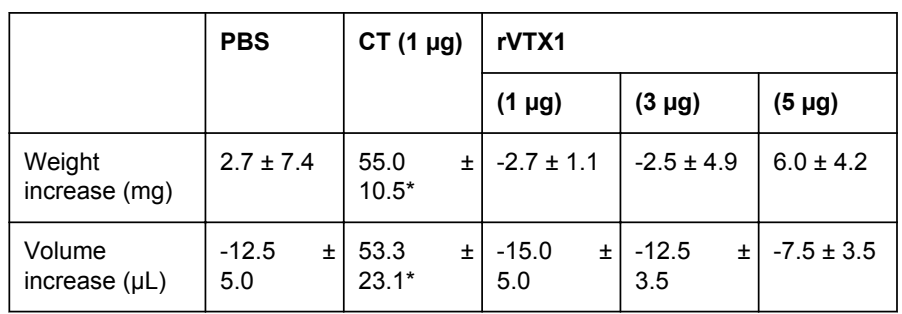

Table 1: Toxicity of rVTX1 in ligated ileal loop in mice. Intestinal ligated ileal loop were practiced in BALB/c mice $(n=3)$ which were inoculated with $50 \mu \mathrm{l}$ of three different concentrations $(1 \mu \mathrm{g}, 3 \mu \mathrm{g}$ or 5 $\mu \mathrm{g})$ of rVTX1. The data correspond with the increase values compared with untreated loops (ileal loop's weight and volume, $3 \mathrm{~h}$ after the inoculation). CT $(1 \mu \mathrm{g})$ was used as positive control, and PBS as negative control. Significant differences between treatments are indicated by asterisks ( ${ }^{*}$ p. 0.05$)$.

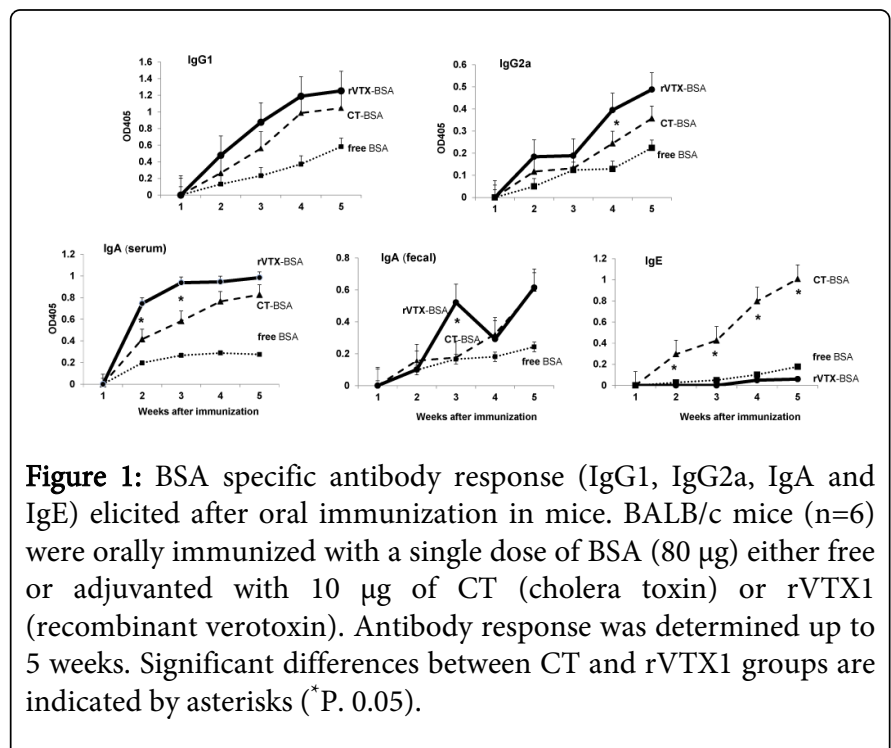

\section{Evaluation of adjuvant activity after oral administration}

In order to study the adjuvant activity of rVTX1, the protein BSA was used as a model antigen. BALB/c mice were orally immunized with a single dose of BSA ( $80 \mu \mathrm{g}$ or $20 \mu \mathrm{g})$ in the presence or absence of the adjuvant CT or rVTX1, and the specific antibody responses evaluated by ELISA. First, we will describe the BSA-specific antibody response, and, later, against the toxoid used as adjuvant.

BSA specific response: The evolution of the serum (IgG1, IgG2a, IgA, IgE) specific antibodies and mucosal (IgA) responses was determined by indirect ELISA. High levels of IgG2a and IgG1 isotypes, were induced after oral immunization with any of the three toxoids. rVTX1 elicited the highest levels and was also the most effective in inducing specific IgA in serum, as well as in mucosal surfaces (Figure 1).There was observed a dose-response effect ( $80 \mu \mathrm{g}$ vs. $20 \mu \mathrm{g}$ ) but with a similar kinetic pattern (not shown). Concerning a potential allergenic effect, IgE levels were also measured in serum (Figure 1). 
Free BSA or co-administered with rVTX1 did not induce a BSAspecific IgE response. In contrast, CT induced the highest levels of IgE in serum, being statistically significant comparing with the rest of the groups.

Toxoid specific response: The neutralization of the adjuvanticity of the toxoids by pre-formed antibodies is a problem that could abrogate the adjuvant effect. Thus, the antibody response elicited against the own toxoid was also measured. The results showed that the toxoid antibody response in sera (IgG1, IgG2a, IgA) or feces (IgA) were low (Figure 2). The highest levels of antibodies IgG1 and IgG2A isotypes (2.A) correspond to the commercial adjuvant CT. There were significant differences in the IgG2a levels between CT and rVTX1.

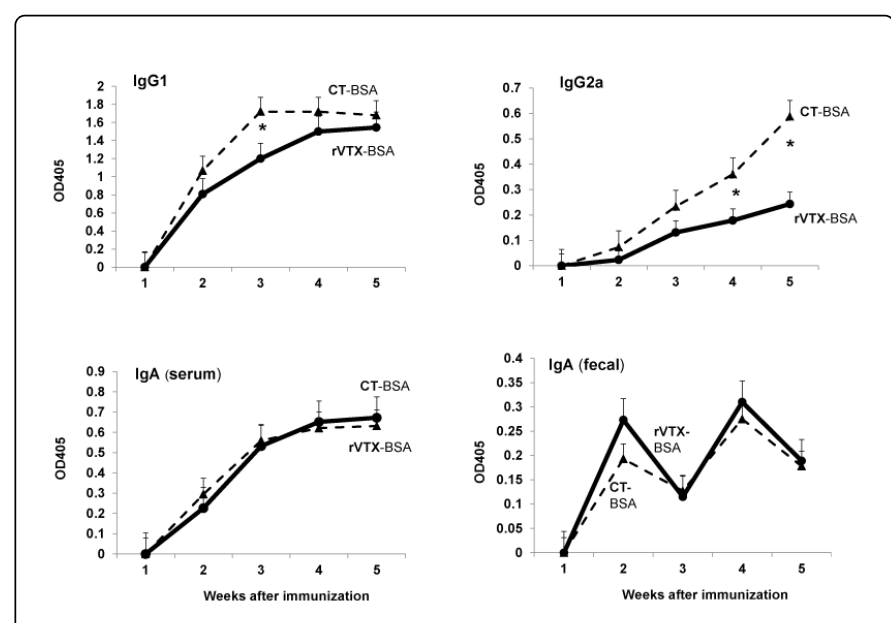

Figure 2: Toxoid specific antibody response (IgG1, IgG2a, IgA) elicited after oral immunization in mice. BALB/c mice $(n=6)$ were orally immunized with a single dose of BSA $(80 \mu \mathrm{g})$ either free or adjuvanted with $10 \mu \mathrm{g}$ of CT (Cholera Toxin) or rVTX1 (recombinant verotoxin). Antibody response against the corresponding protein-adjuvant was determined up to 5 weeks. Significant differences between CT and rVTX1 groups are indicated by asterisks ( ${ }^{*}$ P. 0.05$)$

\section{Discussion}

Vaccines delivered through mucosal surfaces are increasingly studied because of their properties to effectively induce both systemic and mucosal immune responses, as well. In addition, they are cost-less, safer, easily administrable and suitable for mass vaccinations [28]. The main obstacle is to reach the inductive sites of the gut and to break the natural immune tolerance of those mucosal sites. This challenge may be overcome by using adjuvants [29]. Bacterial toxoids, such as CT and LT have been used as oral adjuvants for 20 years. Even though its adjuvanticity, these toxoids present some problems related to toxicity [18] and allergenicity to bystander antigens [2]. Many diverse compounds have been tested as alternative mucosal adjuvants, such as microbial products, cytokines and particulate delivery systems, among others [30]. Bacterial flagellin, being recognized by TLR5 on antigen presenting cells and enterocytes, has been successful used as mucosal adjuvant [3] however, it may be ineffective under certain circumstances [31]. DNA adjuvants, some cytokines and chemokines have also been studied as mucosal adjuvants [4], however, are costly and may induce mutations [3]. Particle delivery systems, such as nanoparticles, have been studied extensively as adjuvants [5,7-9], however, the current high production costs may limit a large-scale production $[4,5]$.

We present here the preliminary results obtained with a new recombinante bacterial toxin (rVTX1) with respect toxicity and oraladjuvanticity. First of all, the absence of toxicity findings after oral inoculated mice (above $40 \mu \mathrm{g}$ ) and in the ligated ileal loop assays (above $5 \mu \mathrm{g}$ ), support the use of rVTX1as adjuvant. In contrast, CT (1 $\mu \mathrm{g})$ induced inflammation in the ligated ileal loops with a signficant luminal fluid accumulation, massive cellular infiltrates and damage in the intestinal villi. In contrast, the intestinal villi of the rVTX1samples appeared with no signs of induced toxicity.

Although the B subunits of the CT and VTX have the same protein fold and function as lectins for glycolipids [24], they differ in the number and structure of the carbohydrates that they recognize: cholera toxin B-pentamer (CT-B) has one binding site per subunit to the branched pentasaccharide of ganglioside GM1, while the verotoxin B-pentamer (VTX-B) has three binding sites per subunit to the trisaccharide portion of globotriaosyl ceramide (Gb3), [32,33]. On the other hand, there are two forms of VTX (VTX1, VTX2) that share structure but with different toxicity, thus, it has been found that VTX-2 is 400 times more toxic (as quantified by LD50 in mice) than VTX-1 [33]. VTX1 consist of an enzymatically active A subunit, and pentamers of B subunits, responsible for their binding to host cells [24]. The SDS-PAGE and immunoblotting analysis of the rVTX1 revealed a subunit A with lower AMW with respect native VTX1 (30 $\mathrm{kDa}$ vs. $31 \mathrm{kDa}$, respectively). Comparing both sequences in gene bank data bases, it was revealed a deletion in the subunit A1 of 6 aminoacids (MHHHHH) equivalent to $820 \mathrm{Da}$, approximately, confirming the 1 $\mathrm{kDa}$ AMW difference. Several mutant exotoxins have been described elsewhere with diminished enterotoxic properties but retaining immunoadjuvant capacity [34]. For instance, it has been reported that minor mutations in the A subunit may reduce its toxicity. Thus, Bosworth et al., by a site directed mutagenesis of the A subunit gene, produced a mutant that differed from the parent toxin in just one aminoacid. This product was safely tested as a potential vaccine demonstrating that the immune response and adjuvant effect induced in mice are not dependent on the toxic activity of the A subunit [35]. Accordingly, we hypothesized that the "toxoid" nature of the here described VTX1-R adjuvant candidate could reside in the observed deletion of subunit A [36,37]. However, further studies must be done to verify this hypothesis.

When rVTX1 was tested as oral adjuvant when co-administered with BSA, strong specific serum and mucosal antibody responses were induced. The levels of IgG1, IgG2a and IgA were even higher than those obtained with CT adjuvant, which have been reported to induce good antibody responses at mucosal surfaces $[3,4]$. The induction of IgG2a, TH1 marker in mice, is very significant as being very difficult to achieve after oral immunization [17].

Results also confirm the previously reported drawback for CT as adjuvant since increases the allergenicity, IgE response, of the coadministered antigen [37]. In contrast, oral rVTX1-BSA inoculation did not elicited specific IgE, adding an extra-value of confidence for the use of this new oral adjuvant candidate.

Concerning the elicited response against the own toxoid, our results indicate that, although rVTX1 elicited antibodies to itself, the levels were similar to those induced by CT against itself but lower in the case of the IgG2a isotype. This is an important concern about the use of immunogenic proteins as adjuvants due to neutralization by pre- 
existing antibodies [38]. This is particularly relevant when the proteinadjuvant is used in several doses and in different vaccines [3]. In any case, the effect of those hypothetical neutralizing properties should be tested.

Finally, due to the lack of toxicitity and immunogenic properties of rVTX1 another prospect is to use this toxoid as a vaccine component against related enterotoxic pathogens, such as Shigella [39].

In summary, the results presented here suggest the feasibility of using rVTX1 as a mucosal adjuvant, since it induces a high antibody response against the co-administered antigen without IgE induction, and has no toxicity under the reported experimental conditions. However, further studies are required in order to assess the lack of toxicity under different host conditions, the resistance to degradation in acidic and surfactant conditions, or the capacity to induce and elicit cellular immune response at the inductive tissues like Peyer patches.

\section{Aknowledgement}

This work was partially funded by the Plan Nacional de Investigación Científica, Desarrollo e Innovación Tecnológica of Spain, co-funded by the European regional development fund, under the grant PI12/01358; and the Spanish Ministerio de Economía y Competitividad, under the grant RTC-2014-2004-2.

\section{References}

1. Kozlowski PA, Cu-Uvin S, Neutra MR, Flanigan TP (1997) Comparison of the oral, rectal, and vaginal immunization routes for induction of antibodies in rectal and genital tract secretions of women. Infect Immun 65: 1387-1394.

2. Neutra MR, Kozlowski PA (2006) Mucosal vaccines: the promise and the challenge. Nat Rev Immunol 6: 148-158.

3. Kunisawa J, Mcghee J, Kiyono H (2007) Mucosal S-IgA enhancement: development of safe and effective mucosal adjuvants and mucosal antigen delivery vehicles. Mucosal Immune Defense: Immunoglobulin A 345-389.

4. Giese M (2013) Molecular Vaccines-From Prophylaxis to Therapy. Medical Microbiology 1: 447.

5. Sijun H, Yong X (2009) Helicobacter pylori vaccine: mucosal adjuvant \& delivery systems. Indian J Med Res 130: 115-124.

6. Nizard M, Diniz MO, Roussel H, Tran T, Ferreira LC, et al. (2014) Mucosal vaccines: novel strategies and applications for the control of pathogens and tumors at mucosal sites. Hum Vaccin Immunother 10: 2175-2187.

7. Gamazo C, Martín-Arbella N, Brotons A, Camacho AI, Irache JM (2015) Mimicking microbial strategies for the design of mucus-permeating nanoparticles for oral immunization. Eur J Pharm Biopharm .

8. Irache JM, Salman HH, Gamazo C, Espuelas S (2008) Mannose-targeted systems for the delivery of therapeutics. Expert Opin Drug Deliv 5: 703-724.

9. Salman HH, Gamazo C, Campanero MA, Irache JM (2005) Salmonellalike bioadhesive nanoparticles. J Control Release 106: 1-13.

10. Holmgren J, Czerkinsky C (2005) Mucosal immunity and vaccines. Nat Med 11: S45-53.

11. Charles RC, Hilaire IJ, Mayo-Smith LM, Teng JE, Jerome JG, et al. (2014) Immunogenicity of a killed bivalent (O1 and O139) whole cell oral cholera vaccine, Shanchol, in Haiti. PLoS Negl Trop Dis 8: e2828.

12. Matoba N, Geyer BC, Kilbourne J, Alfsen A, Bomsel M, et al. (2006) Humoral immune responses by prime-boost heterologous route immunizations with CTB-MPR(649-684), a mucosal subunit HIV/AIDS vaccine candidate. Vaccine 24: 5047-5055.

13. Lv X, Yang J, Song H, Li T, Guo L, et al. (2014) Therapeutic efficacy of the multi-592 epitope vaccine ctb-ue against helicobacter pylori infection in a mongolian gerbil model and its microrna-155-associated immunoprotective mechanism. Vaccine 32: 5343-5352.

14. Salazar-Gonzalez JA, Rosales-Mendoza S, Romero-Maldonado A, Monreal-Escalante E, Uresti-Rivera EE, et al. (2014) Production of a plant-derived immunogenic protein targeting apob100 and cetp: Toward a plant-based atherosclerosis vaccine. Mol Biotechnol 56: 1133-1142.

15. Sun JB, Czerkinsky C, Holmgren J (2010) Mucosally induced immunological tolerance, regulatory $\mathrm{T}$ cells and the adjuvant effect by cholera toxin B subunit. Scand J Immunol 71: 1-11.

16. Lebens M, Holmgren J (1994) Mucosal vaccines based on the use of cholera toxin B subunit as immunogen and antigen carrier. Dev Biol Stand 82: 215-227.

17. Lycke N, Holmgren J (1986) Strong adjuvant properties of cholera toxin on gut mucosal immune responses to orally presented antigens. Immunology 59: 301-308.

18. Sanchez J, Holmgren J (2011) Cholera toxin - a foe \& a friend. Indian J Med Res 133: 153-163.

19. Kubota E, Joh T, Tanida S, Sasaki M, Kataoka H, et al. (2005) Oral vaccination against Helicobacter pylori with recombinant cholera toxin B-subunit. Helicobacter 10: 345-352.

20. Sánchez J, Holmgren J (2008) Cholera toxin structure, gene regulation and pathophysiological and immunological aspects. Cell Mol Life Sci 65: 1347-1360.

21. Holmgren J, Czerkinsky C, Lycke N, Svennerholm AM (1994) Strategies for the induction of immune responses at mucosal surfaces making use of cholera toxin B subunit as immunogen, carrier, and adjuvant. Am J Trop Med Hyg 50: 42-54.

22. Brereton CF, Sutton CE, Ross PJ, Iwakura Y, Pizza M (2011) Escherichia coli heat-labile enterotoxin promotes protective Th17 responses against infection by driving innate IL-1 and IL-23 production. J Immunol 186: 5896-5906.

23. Gupta RK, Relyveld EH, Lindblad EB, Bizzini B, Ben-Efraim S, et al. (1993) Adjuvants--a balance between toxicity and adjuvanticity. Vaccine 11:293-306

24. Beddoe T, Paton AW, Le Nours J, Rossjohn J, Paton JC (2010) Structure, biological functions and applications of the AB5 toxins. Trends Biochem Sci 35: 411-418.

25. Karmali MA, Petric M, Lim C, Cheung R, Arbus GS (1985) Sensitive method for detecting low numbers of verotoxin-producing Escherichia coli in mixed cultures by use of colony sweeps and polymyxin extraction of verotoxin. J Clin Microbiol 22: 614-619.

26. Matsushiro A, Sato K, Miyamoto H, Yamamura T, Honda T (1999) Induction of prophages of enterohemorrhagic Escherichia coli O157:H7 with norfloxacin. J Bacteriol 181: 2257-2260.

27. Camacho AI, Souza-Rebouças J, Irache JM, Gamazo C (2013) Towards a non-living vaccine against Shigella flexneri: from the inactivation procedure to protection studies. Methods 60: 264-268.

28. Petrovsky N, Aguilar JC (2004) Vaccine adjuvants: current state and future trends. Immunol Cell Biol 82: 488-496.

29. Junqueira-Kipnis AP, Marques Neto LM1, Kipnis A (2014) Role of Fused Mycobacterium tuberculosis Immunogens and Adjuvants in Modern Tuberculosis Vaccines. Front immunol 5: 188.

30. Mizel SB, Bates JT (2010) Flagellin as an adjuvant: cellular mechanisms and potential. J Immunol 185: 5677-5682.

31. Shamini G, Ravichandran M, Sinnott JT, Somboonwit C, Sidhu HS, et al. (2011) Structural inferences for Cholera toxin mutations in Vibrio cholerae. Bioinformation 6: 1-9.

32. Fuller CA, Pellino CA, Flagler MJ, Strasser JE, Weiss AA (2011) Shiga toxin subtypes display dramatic differences in potency. Infect Immun 79: 1329-1337.

33. Del Giudice G, Rappuoli R (1999) Genetically derived toxoids for use as vaccines and adjuvants. Vaccine 17 Suppl 2: S44-52.

34. Bosworth BT, Samuel JE, Moon HW, O'Brien AD, Gordon VM, et al. (1996) Vaccination with genetically modified Shiga-like toxin IIe prevents edema disease in swine. Infect Immun 64: 55-60. 
Citation: Matías J, Landeta O, Esquivias P, Gamazo C (2015) Preliminary Studies on a Derivative Verotoxin as Oral Adjuvant. J Vaccines Vaccin 6: 279. doi:10.4172/2157-7560.1000279

Page 6 of 6

35. Pizza M, Giuliani MM, Fontana MR, Monaci E, Douce G, et al. (2001) Mucosal vaccines: non toxic derivatives of LT and CT as mucosa adjuvants. Vaccine 19: 2534-2541.

36. Nawar HF, Arce S, Russell MW, Connell TD (2005) Mucosal adjuvant properties of mutant LT-IIa and LT-IIb enterotoxins that exhibit altered ganglioside-binding activities. Infect Immun 73: 1330-1342.

37. Shimizu T, Hamabata T, Yoshiki A, Hori T, Ito S, et al. (2003) An association of $27-$ and $40-\mathrm{kDa}$ molecules with glycolipids that bind A-B bacterial enterotoxins to cultured cells. Biochim Biophys Acta 1612: 186-194.
38. McCoy K, Tatsis N, Korioth-Schmitz B, Lasaro MO, Hensley SE, et al. (2007) Effect of preexisting immunity to adenovirus human serotype 5 antigens on the immune responses of nonhuman primates to vaccine regimens based on human- or chimpanzee-derived adenovirus vectors. J Virol 81: 6594-6604.

39. Camacho AI, Irache JM, Gamazo C (2013) Recent progress towards development of a Shigella vaccine. Expert Rev Vaccines 12: 43-55. 